\title{
A Comprehensive Approach towards Extra-Corporal Circulation Control using Fuzzy Logic
}

\author{
Benedikt Baumgartner, Alejandro Mendoza, Ulrich Schreiber, Stefan Eichhorn, Markus Krane, \\ Robert Bauernschmitt and Alois Knoll
}

\begin{abstract}
Extra-corporal Circulation Support Systems (ECCS) are used in cardiac surgery on a daily basis. Surgeons and perfusionists supervise patients' vital signals such as heart rate or blood pressure and ensure secure and errorless operation of the system. An automated ECCS can help to reduce the workload of human operators and can provide a patient with optimal perfusion. In our opinion a fuzzy controller is predestinated for autonomus heart-lung machine (HLM) control, since it allows a straightforward implementation of expert knowledge and is able to deal with inherently imprecise data. In this work we present a comprehensive approach towards intelligent HLM automation that uses fuzzy logic as a control methodology. This approach includes the development of a mechanical circulatory model, a purely virtual model, as well as animal experiments.
\end{abstract}

\section{INTRODUCTION}

Fuzzy control is a well-established method in control engineering and has been applied to a wide range of problems in diverse disciplines. It is especially useful if a direct analytical description of the control process seems unsuitable. Since fuzzy logic is able to interpret vague and subjective knowledge and allows qualitative system descriptions it is highly qualified for medical decision making. In the following we apply fuzzy logic to a real-world control problem, the automation of a portable HLM. We believe, that fuzzy control is an appealing technique for this task.

\section{A. Background}

Heart-lung-machines (HLM) are indispensable in today's cardiac surgery. When used in an operating theater expert knowledge is always on-hand. The HLM and the patient are constantly supervised by surgeons, perfusionists and anesthesiologists. However, patients suffering from cardiogenic shock would benefit from an early application of an ECSS, preventing multi organ failure. Latest developments clear the way to use an ECCS for emergency circulatory resuscitation in non-clinical environments, even when trained staff is sparse and when there is no standard monitoring setup. An automated ECCS can help to guarantee optimal perfusion of the patient while minimizing the workload of a human operator in stressful situations. The goal of this work is to develop a self-contained system that is able

Benedikt Baumgartner, Alejandro Mendoza and Alois Knoll are with the Department of Informatics, Technische Universität München, Boltzmannstr. 3, 85748 Garching, Germany (phone: +49 89289 18106; email: \{baumgarb, mendozag, knoll\}@in.tum.de ).

Ulrich Schreiber, Stefan Eichhorn, Markus Krane and Robert Bauernschmitt are with the German Heart Center Munich, Technische Universität München, Lazarettstr. 36, 80636 München, Germany (phone: +49 89 12180; email: \{schreiberu, eichhorn, krane, bauernschmitt\}@dhm.mhn.de ). to regulate perfusion based on online data of the patient. Because of the application in a non-clinical environment special requirements have to be fullfilled: the control system has to be highly dynamic, so that it can adapt to different situations and different needs of the patient. At the same time it must be robust and patient safety must be ensured at all times.

We clarify why fuzzy control is an appealing technique for this task but also discuss its difficulties. Furthermore, we present a holistic approach that eases the development of a HLM's fuzzy controller. This approach includes 4 steps: Data acquisition in animal experiments, development of a mechanical circulatory model, establishment of a virtual development platform and control evaluation. This work is organized as follows: First we explain why animal experiments are essential for the success of the project. We show how the development of a mechanical system model, as well as a virtual model influence the controller design and subsequently present results that verify our models and justify the use of a fuzzy controller.

\section{B. Related Work}

The non-trivial task of extra-corporal circulation control has already been adressed in numerous publications. Back in 1978 Prilutskii et al. considered design principles for an automatic ECC [1] and identified four linked subsystems relevant to circulation control: hemodynamic body parameters, oxygenation parameters, cardiovascular and temperature parameters. Rao et al. developed a model predictive controller to regulate hemodynamic variables in critical care subjects and revealed that automatic control outperformed manual regulation [2]. Misgeld et al. compared three different control schemes to regulate blood flow [3]. A proportional plus integral (PI) controller, a self-tuning general predictive controller (GPC) and a $H_{\infty}$ which showed the best control performance during tests in a circulatory simulator. Boschetti et al. developed an ECC simulation that can be used to identify control strategies [4] that consists of a hemodynamic model, a heat exchange model and a gas transfer model. Meyrowitz developed a prototype of an automatically controlled HLM [5] using PID and predictive controllers.

Fuzzy logic has also been applied to various medical control problems. E.g. fuzzy modelling was used to monitor depth of anaesthesia [6]. Bauernschmitt et al. used fuzzy control to regulate hemodynamics [7]. Bates and Young give a comprehensive overview of fuzzy decision making systems in the critical care unit [8]. Many more examples 
could be given. Fuzzy systems seem to be popular in the medical domain since they are able to handle vague data and the design process based on natural language allows a straightforward implementation of expert knowledge, in particular from medical practitioners.

\section{Animal Experiments}

Animal experiments are essential in order to build up a realistic database of extra-corporal perfusion parameters. They help to identify parameters in different stages of an operation depending on the patient's constitution as well as on human interventions such as drug administration. Furthermore they are essential in the evaluation stage, when fuzzy logic takes control over the HLM.

For experiments we use ordinary pigs of approximately $80 \mathrm{~kg}$. The experiments were approved by the Government of Upper Bavaria. As an ECCS the Lifebridge B2T [9] was used. It is a portable, modular and easy to use ECCS, designed for emergency transportation or resuscitation. Machine parameters, i. e. pump speed (revolutions per minute, $\mathrm{rpm}$ ), flow (liters per minute, lpm), inlet, outlet and pressure before oxygenation (all in $\mathrm{mmHg}$ ) are recorded via a CAN Bus listener. The pump speed can also be controlled remotely via serial communication.

Physiological data from the patient are recorded with a tailor-made software that allows the integration of different sensor interfaces. In this framework we record standard ECG, blood pressures (mean arterial and central venous), blood flow, $\mathrm{SpO} 2$ as well as different blood gases acquired from a CDI 500 online monitor. Our software allows the recording and visualization of the patient and machine data in a consistent way. The sampling frequency is $200 \mathrm{~Hz}$.

Animal experiments were conducted following a strict protocol. Among others steady perfusion, tube kinking, lifting and lowering of the ECCS, ventricular fibrillation, mechanical movements of sensor devices and the ECCS and increase and decrease of centrifugal pump speed were examined. Figure 1 shows our setup in a schematic way. The patient (pig) is cannulated with the Lifebridge HLM via the femoral artery and vein and different sensor data is acquired. The collected data is recorded for offline analysis and simultaneously made available in a local network. Client programs such as patient monitors or a control software can access the data streams.

\section{Hydraulic Model}

A hydraulic blood circuit model allows to simulate hemodynamics in a standardized setting. Different control schemes can be tested and possible errors can be eliminated before testing a controller in an animal experiment. For a mechanical model of the circulatory system we follow the socalled 'Westerhof model', elaborately described by Sharp and Dharmalingam [10]. It consists of two resistances (R) and one compliance element $(\mathrm{C})$ in a RCR configuration.

Figure 2 shows our model configuration in detail. The Arterial Cannula Resistance (ACR) represents the cannula for the femoral artery and Venous Cannula Resistance (VCR)

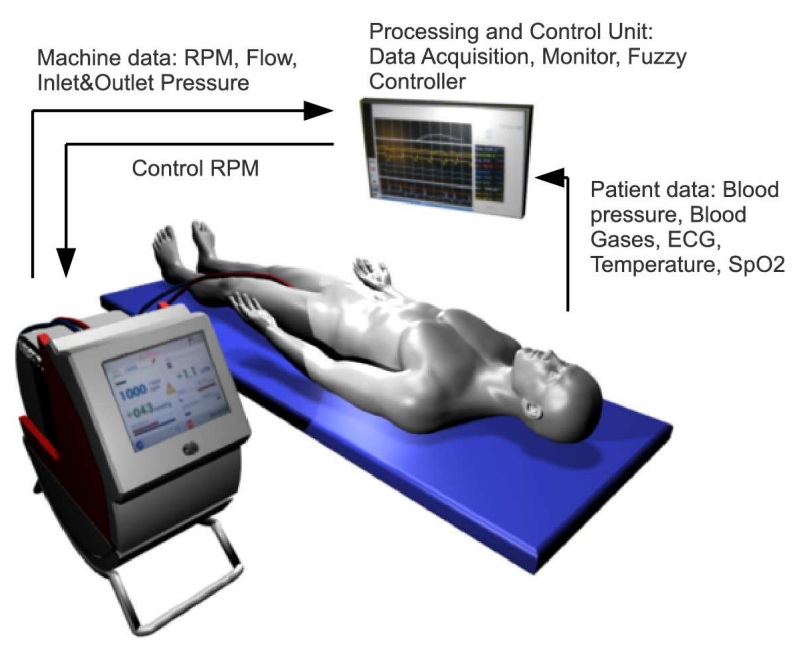

Fig. 1. Schematic experiment setup

for the femoral vein. The R1, C, R2 elements represent the organism itself which comprises of an initial resistance (R1), a compliance (C) and an outlet resistance (R2) of the blood circuit. Both resistances are variable and can be altered with electromechanical driven pistons. In the hydraulic blood circuit model also a beating heart was implemented. Using a roller pump (Ismatec BVP) different heart rates can be adjusted. The Arterial Flow Sensor (AFS) detects the inner arterial flow of the ECCS and additionally the centrifugal pump speed (rpm) is acquired. With the arterial pressure sensor (APS) the relevant mean arterial pressure (MAP) is detected. A concise description of the mechanical mock model can be found in [11].

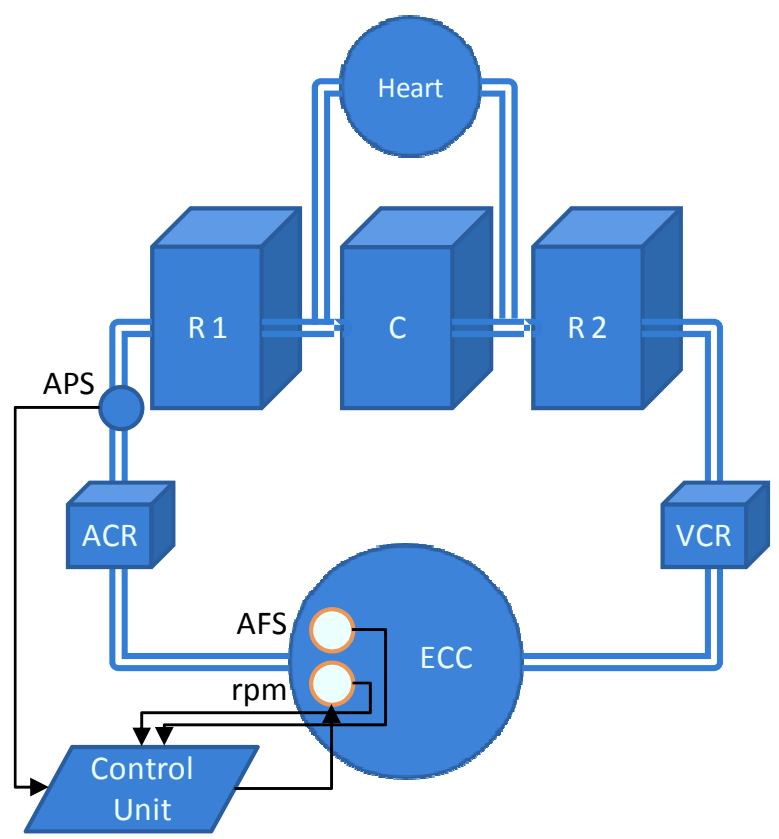

Fig. 2. Hydraulic model setup 


\section{E. Virtual Model}

A virtual model of both the patient and the ECCS allows to extensively test control strategies. Furthermore, specific situations such as drug administration or tube kinking can be easily modeled and evaluated. There exist quite many models of the human circulatory system. For our purpose we chose a closed loop cardiopulmonary system composed of a fourchamber varying-elastance heart, a pericardium, a systemic circulation, and a pulmonary circulation. The basic model and the simulation software JSim can be obtained from the Physiome project [12], [13]. A model of the ECCS was designed and integrated with the simulation environment. Therefore the main components of the Lifebridge HLM - a reservoir, a centrifugal pump, an oxygenator, a filter, tubing, and cannulas - were described in a mathematical modeling language. Schematics for the cardiovascular model as well as the ECCS model are shown in Figure 3. Details on both virtual models can be found in [14].

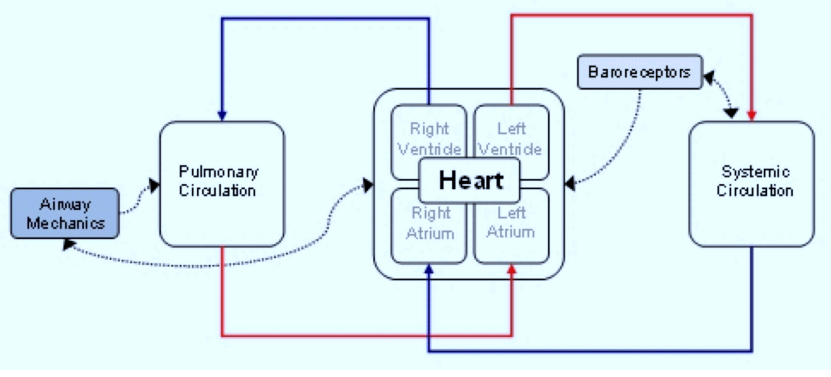

(a)

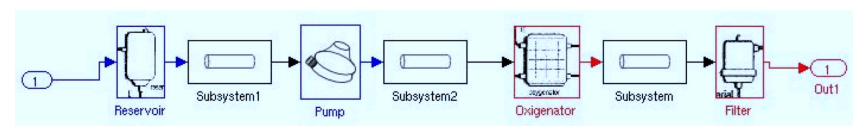

(b)

Fig. 3. (a) shows the cardiovascular model obtained from the Physiome project. (b) displays the components of the Lifebridge HLM used in the ECCS model.

\section{F. Fuzzy Controller}

We use a standard fuzzy system with Mamdani inference to control the pump speed of the HLM. Our controller focuses on hemodynamic regulation. Oxygenation, temperature or blood gas regulation is beyond focus of this work. The input parameters of our control loop are the MAP and the flow produced by the ECCS. Obviously these parameters have a major influence on perfusion [15]. For the input variables 3 sets are defined, labled as low, medium and high. The controller output is a correction factor of the current pump speed (Delta_RPM). The fuzzy sets were designed with the help of heart surgeons and perfusionists and represent their knowledge and experience. The rulebase is intuitive: If the MAP or flow are low, the pump speed is increased. If the inputs are in a medium range, the speed is steady and if the parameters indicate high values, the pump speed is decreased. The fuzzy sets for both input and output are displayed in
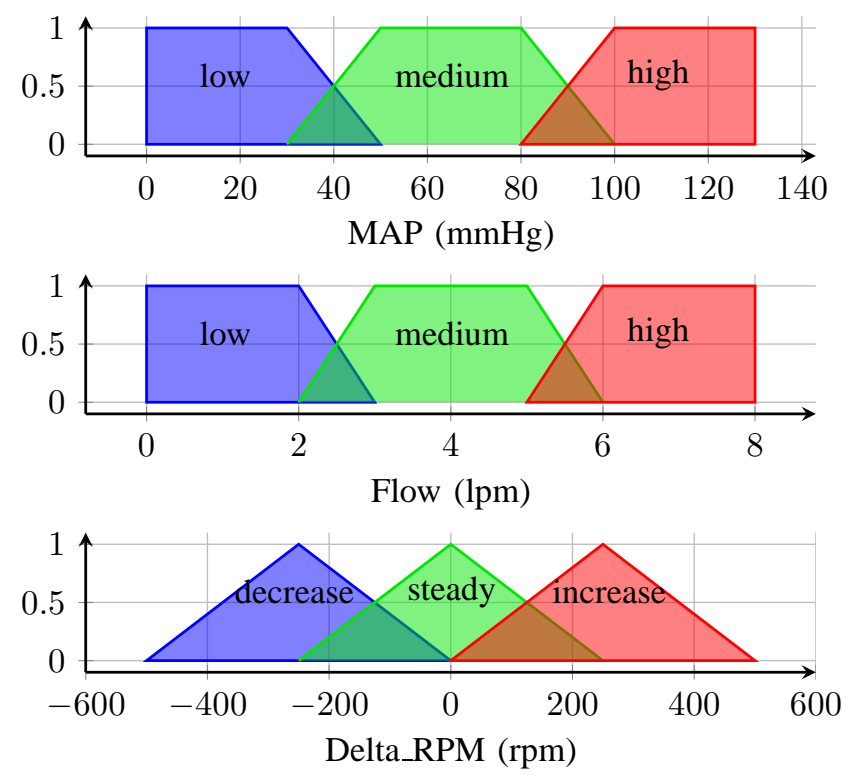

Fig. 4. From top to bottom the fuzzy sets for the controller's input parameters MAP and produced pump flow as well as the output set for pump speed regulation are shown.

Figure 4. From a technical point of view the controller accesses the input data as a client over the local network. The output is sent to the ECCS via serial connection. The controller operates at $0.5 \mathrm{~Hz}$, i.e. every two seconds the current values for MAP and flow are obtained and a new pump speed is set according to the controller's output. During animal experiments users can switch between automatic and manual control at any time. Our integral approach and software design allows consistent data recording and control evaluation in our virtual and hydraulic model and in animal experiments.

\section{MAin Results}

Illustratively we show 2 graphs obtained during an animal experiment. $0.1 \mathrm{mg}$ of Arterenol, a vasoconstrictor, was administered. The top plot in Figure 5 displays the increase in MAP caused by the medication. Simultaneously the controller reacts with a decrease of Delta_RPM, the correction factor of the pump speed. Looking at the nominal (RPM_nom) and actual (RPM_act) pump speed, we see a smooth decrease as expected. After about 20 seconds a decrease in the MAP due to pump speed reduction is observed. Figure 6 illustrates just the opposite behaviour. We administered $2 \mathrm{mg}$ of the vasodilator ISD. Its effect is visible in the MAP drop. However, the controller starts to counteract the medication with a pump speed increase. Both experiments show smooth control characteristics without overshoots or oscillations. The step-like characteristics of the nominal speed curves have two main reasons: first, the controller does not constantly adapt the speed but only every 2 seconds as described above. Second, the HLM only allows changes at a step-size of $50 \mathrm{rpm}$. Differences between the actual and nominal curve are due to system properties such 

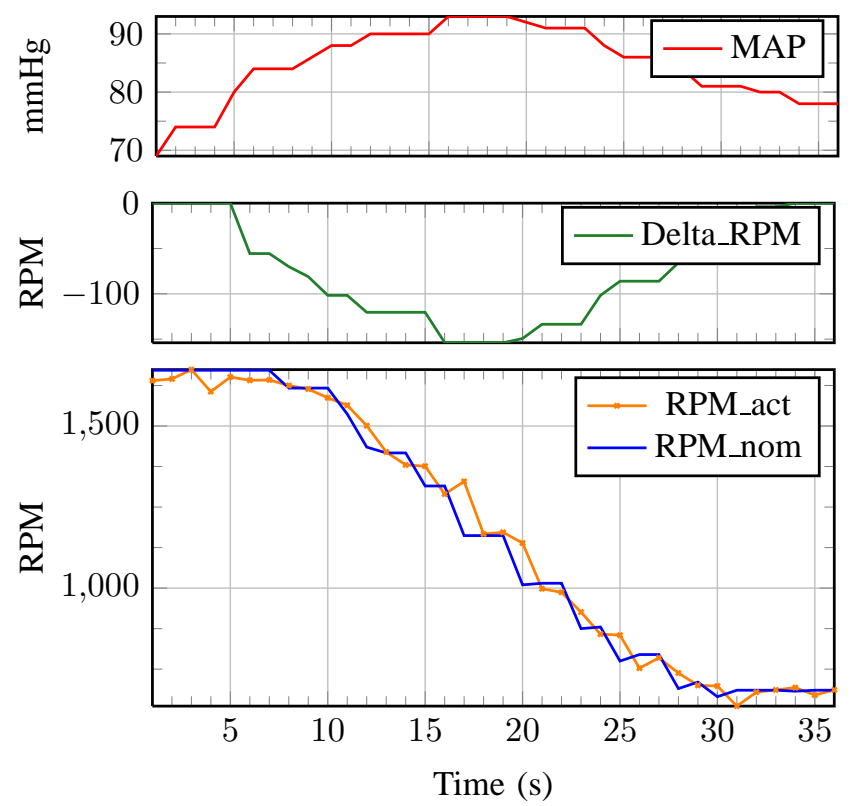

Fig. 5. Vasokonstriktion. From top to bottom the diagrams show the MAP, the controller's output (Delta_RPM) the nominal and current pump speed (RPM_nom, RPM_act) of the Lifebridge ECCS.

as friction.

Concerning the hydraulic and virtual models, similar behavior can be observed. In the mechanical setup the resistances were tuned such that the initial MAP was either small $(30 \mathrm{mmHg})$ or big $(90 \mathrm{mmHg})$. The fuzzy controller reacted with an increase and decrease of the pump speed respectively, so that the pressure and the flow were in a medium range again. Details can be found in [11]. The virtual model was verified by comparing its results with real experiment data. Figure 7 shows the pump speed of the ECCS in relation to its produced flow. During animal experiments the pump was set to specific speeds and the produced flow was recorded. For the sake of clarity, we only display data from one experiment (A). The hydraulic model $(\mathrm{H})$ was tuned to resemble the average of several animal experiments. For the virtual model (V) a second-order approximation was sufficient to model the ECCS.

Tests have shown, that the controller exhibits smoother regulation when mean values of MAP and flow are considered instead of the sometimes noisy online data. Also system latencies play an important role in this application. Since hemodynamics and general consitution of a patient is different from one to each other, they react differently to medical interventions and therefore differently to extracorporal perfusion and changes of the pump speed.

\section{CONCLUSIONS}

We have presented a comprehensive approach towards extra-corporal circulation control. This approach has 3 main components. Animal experiments, a purely virtual and a hydraulic circulatory model. Animal experiments are essential to have ground truth data and to evaluate control behavior.
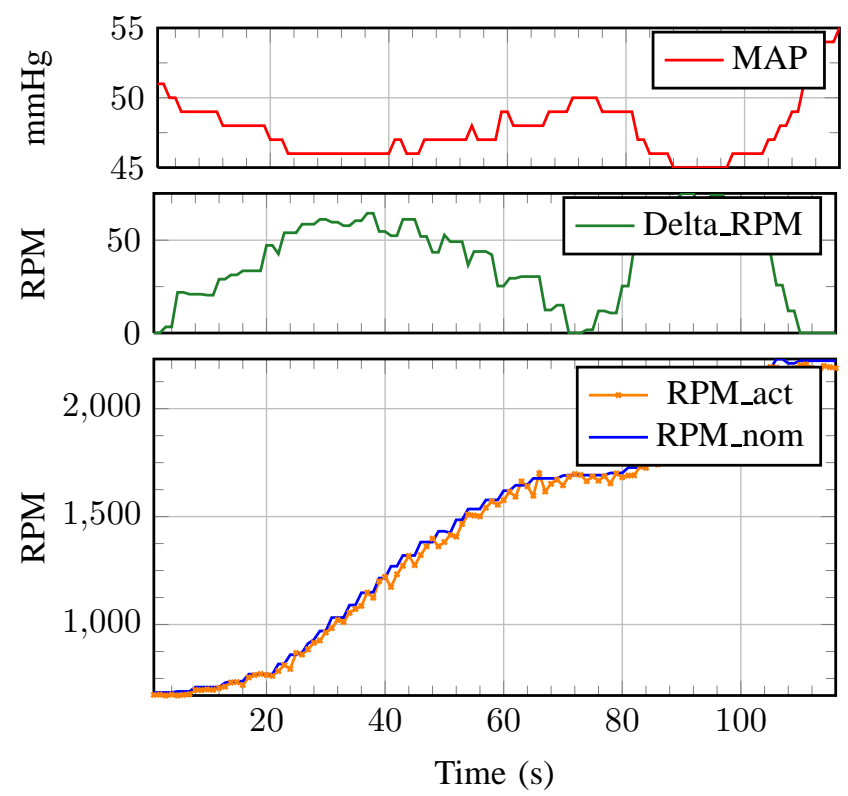

Fig. 6. Vasodilatation. See Figure 5

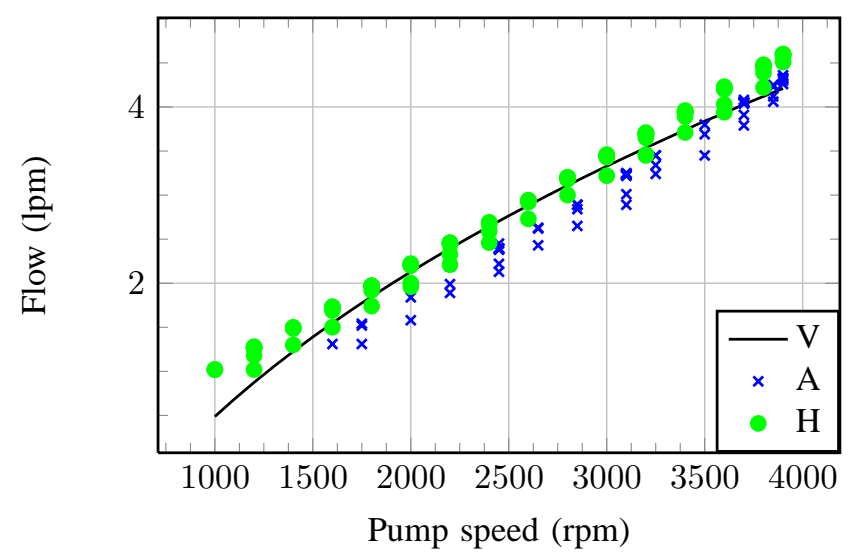

Fig. 7. Comparison of animal experiment data (A) with hydraulic $(\mathrm{H})$ and virtual (V) model.

The virtual model helps to easily design controllers and to compare them in a standardized and reproducible way. The hydraulic model helps to identify possible hard- or software errors before animal experiments. We implemented a basic fuzzy controller with two input and one output variables. We showed, how the controller reacts to medication during an animal experiment.

Fuzzy logic was found to be well-suited for perfusion control, since a direct, analytical description of the control process is unsuitable. Hard limits for hemodynamic variables are improper. Furthermore, manually controlled perfusion relies on evidence-based guidelines as well as on the personal experience of the operator. We believe that fuzzy logic is therefore predestinated for this task as our results confirm.

In future work we will compare the results of fuzzy control to classical control methodologies such as PID or ModelPredictive Control to foster our assumptions. 


\section{ACKNOWLEDGMENT}

This work was supported by the Graduate Scool for Information Science in Health (GSISH) and by an unrestricted educational grant from the Bayerische Forschungsstiftung.

\section{REFERENCES}

[1] V. Prilutskii, S. Tropskii, V. Khrenov, and Y. Slavyak, "Analysis of control systems for extracorporeal-circulation equipment," Biomedical Engineering, vol. 12, no. 4, pp. 196-199, Jul. 1978.

[2] R. R. Rao, B. Aufderheide, and B. W. Bequette, "Experimental studies on multiple-model predictive control for automated regulation of hemodynamic variables," IEEE Transactions on Biomedical Engineering, vol. 50, pp. 277-288, 2003.

[3] B. Misgeld, J. Werner, and M. Hexamer, "Robust and self-tuning blood flow control during extracorporeal circulation in the presence of system parameter uncertainties," Medical and Biological Engineering and Computing, vol. 43, no. 5, pp. 589-598, Oct. 2005.

[4] F. Boschetti, S. Mantero, F. Miglietta, M. L. Costantino, F. Montevecchi, and R. Fumero, "An approach to computer automation of the extracorporeal circulation," Computers in Biology and Medicine, vol. 32, no. 2, pp. 73-83, Mar. 2002.

[5] G. Meyrowitz, "Automatisierung der Herz-Lungen-Maschine," Ph.D. dissertation, Universität Karlsruhe, 2005.

[6] D. A. Linkens, J. S. Shieh, and J. E. Peacock, "Hierarchical fuzzy modelling for monitoring depth of anaesthesia," Fuzzy Sets and Systems, vol. 79, no. 1, pp. 43-57, Apr. 1996.

[7] R. Bauernschmitt, J. Hoerer, E. Schirmbeck, H. Keil, G. Schrott, A. Knoll, and R. Lange, "Fuzzy-logic based automatic control of hemodynamics," Computers in Cardiology, vol. 30, pp. 773-776, 2003.

[8] J. H. T. Bates and M. P. Young, "Applying fuzzy logic to medical decision making in the intensive care unit," Am. J. Respir. Crit. Care Med., vol. 167, no. 7, pp. 948-952, Apr. 2003.

[9] M. Krane, D. Mazzitelli, U. Schreiber, A. Mendoza Garzia, B. Voss, C. Badiu, R. Lange, and R. Bauernschmitt, "First experience with a new portable cardiopulmonary bypass system - lifebridge b2t with percutaneous femoral cannulation," in Computers in Cardiology, vol. 35, 2008.

[10] M. Sharp and R. Dharmalingam, "Development of a hydraulic model of the human systemic circulation," ASAIO, vol. 45, no. 6, pp. 535540, 1999.

[11] U. Schreiber, S. Eichhorn, A. Mendoza, B. Baumgartner, R. Bauernschmitt, R. Lange, A. Knoll, and M. Krane, "A new fuzzy controlled extracorporeal circulation system. first results of an in-vitro investigation," in Computers in Cardiology, vol. 36, 2009.

[12] J. B. Bassingthwaighte, "http://nsr.bioeng.washington.edu/."

[13] J. B. Bassingthwaighte, "Strategies for the physiome project," Annals of Biomedical Engineering, vol. 28, no. 8, pp. 1043-1058, 082000.

[14] A. Mendoza García, B. Baumgartner, U. Schreiber, M. Krane, A. Knoll, and R. Bauernschmitt, "Simulation of extracorporal circulation for the design of fuzzy controlled perfusion," in 7th IASTED International Conference on Biomedical Engineering, 2010.

[15] G. S. Murphy, I. Hessel, Eugene A., and R. C. Groom, "Optimal perfusion during cardiopulmonary bypass: An evidence-based approach,' Anesth Analg, vol. 108, no. 5, pp. 1394-1417, May 2009. 